\title{
Germanica
}

\section{Michael Opitz, Wolfgang Hilbig. Eine Biographie}

Frankfurt a.M., Fischer, 2017, 663 p.

\section{Bénédicte Terrisse}

\section{OpenEdition}

\section{Journals}

Édition électronique

URL : http://journals.openedition.org/germanica/4131

DOI : $10.4000 /$ germanica. 4131

ISSN : 2107-0784

Éditeur

Université de Lille

Édition imprimée

Date de publication : 31 décembre 2017

Pagination : 133-141

ISBN : 9782913857407

ISSN : 0984-2632

\section{Référence électronique}

Bénédicte Terrisse, « Michael Opitz, Wolfgang Hilbig. Eine Biographie », Germanica [En ligne], 61 | 2017, mis en ligne le 01 janvier 2021, consulté le 02 mars 2021. URL : http://journals.openedition.org/ germanica/4131 ; DOl : https://doi.org/10.4000/germanica.4131 


\section{Hilbig-Matériau. Vie et œuvre de Wolfgang Hilbig sous le signe de l'archive}

À propos de : Michael Opitz, Wolfgang Hilbig. Eine Biographie, Frankfurt a.M., Fischer, 2017, 663 p.

Annoncée pour le dixième anniversaire de la mort de l'écrivain, la biographie de Wolfgang Hilbig (1941-2007) par Michael Opitz chez Fischer était attendue ${ }^{1}$.

On disposait certes déjà de deux biographies, l'une de Karen Lohse, parue en 2008, et l'autre de Birgit Dahlke, en 20112 - en raison de sa contribution majeure au progrès des connaissances sur l'auteur, cette dernière avait été très chaleureusement accueillie par la communauté des amateurs de l'œuvre de Wolfgang Hilbig33-, cependant leur petit format (respectivement 144 et 143 pages) avait eu davantage pour effet d'aiguiser la curiosité pour l'écrivain que de l'assouvir entièrement. Par ailleurs, étant toutes les deux parues dans de petites maisons d'édition (Plöttner Verlag et Wehrhahn Verlag), ces biographies n'avaient ni reçu l'attention qu'un tel travail méritait, ni, par conséquent, rempli la mission de canonisation implicitement attribuée au genre. L'ouvrage qui vient de paraître dans la maison d'édition qui a publié quasiment toute l'œuvre de Wolfgang Hilbig, en possède les archives et en détient les droits apparaît de ce fait comme la biographie officielle. Son ampleur (663 pages) semble indiquer que Michael Opitz souscrit à ce que François Dosse nomme la « contrainte d'exhaustivité 4 » et que le volume apporte ouvertement sa pierre à l'édifice de canonisation de l'auteur. Il prend place aux côtés des autres ouvrages imposants que représente chacun des six tomes des Hilbig-Werke publiés par les éditions Fischer depuis 2008. Le septième et dernier, qui contient les essais, discours et interviews, doit paraître au printemps 2018.

1. - Michael Opitz, Wolfgang Hilbig. Eine Biographie, Frankfurt a. M., Fischer, 2017, 663 p.

2. - Karen Lohse, Wolfgang Hilbig. Eine motivische Biografie, Leipzig, Plöttner Verlag, 2008 ; Birgit Dahlke, Wolfgang Hilbig, Hannover, Wehrhahn Verlag, 2011.

3. - Heribert Tommek, « Teilnehmende Beobachtung. Ein neues Autorenporträt von Wolfgang Hilbig. (Rezension über: Birgit Dahlke: Wolfgang Hilbig. Hannover: Wehrhahn 2011.) », IASLonline [06.02.2012] URL : http://www.iaslonline.de/index. php? vorgang_id=3490 consulté le 17 novembre 2017.

4. - François Dosse, « Préface. L'explosion biographique », in : Vincent Broqua et Guillaume Marche (dir.), L'épuisement du biographique ?, Cambridge, Cambridge Scholar Publishing, 2010, p. xi-xvi, ici p. xiii. 
L'auteur de la biographie, Michael Opitz, est un habitué des projets d'envergure et des ouvrages de référence puisqu'il a notamment coédité l'encyclopédie sur les notions de Walter Benjamin (Benjamin-Begriffe) (2000), le dictionnaire Metzler de la littérature est-allemande (Metzler Lexikon DDR-Literatur. Autoren - Institutionen - Debatten, 2009, dans lequel il signe l'entrée «Wolfgang Hilbig ») et l'anthologie de poésie In diesem Land. Gedichte aus den Jahren 1990-2010 (2010). Depuis 2008, il a publié plusieurs articles scientifiques sur Hilbig.

La fascination qu'exerce la figure de Wolfgang Hilbig, mais aussi l'énigme que représente son œuvre et que les biographies, témoignages et romans ${ }^{5}$ ne cessent d'interroger sans jamais parvenir à sa résolution, en font un objet de prédilection pour le genre biographique : comment cet écrivain autodidacte de RDA, qui a grandi dans un milieu ouvrier sans livre et quitté l'école à quatorze ans pour exercer différents métiers d'ouvrier, peut-il produire des textes qui rivalisent avec ceux des plus grands poètes et écrivains de la modernité ? C'est ainsi le hiatus entre la vie et l'œuvre qui, dans le cas de Hilbig, suscite la curiosité et l'écriture biographiques.

La biographie de Michael Opitz ne résout pas le mystère, mais elle éclaire de manière décisive cette vie singulière. Organisé en six parties comprenant cinq chapitres chacune, l'ouvrage progresse de manière chronologique et thématique, alternant récit de la vie et présentation des textes. Il comprend aussi de nombreuses photographies en noir et blanc, ainsi qu'une chronologie de la vie de l'écrivain en fin d'ouvrage. Les titres de chaque partie et chapitre sont tirés de citations glanées dans les notes, correspondances ou brouillons de l'auteur.

\section{Sous le signe de l'archive}

À la différence des deux autres biographies qui se fondent en grande partie sur des entretiens, le présent ouvrage se place principalement sous l'égide de l'archive. Si Michael Opitz a rassemblé entre les pages d'un même livre et réordonné le savoir dispersé dans les biographies, témoignages et interviews déjà existants, il a surtout eu accès à un grand nombre de sources inédites, notamment les journaux intimes de Hilbig et sa correspondance avec ses compagnes. Opitz a ainsi exploité le fonds et la collection « Wolfgang Hilbig », mais aussi les fonds « Franz Fühmann » et «Gert Neumann » conservés tous les quatre à l'Akademie der Künste, ceux de «Stephan Hermlin » et « Hans Magnus Enzensberger »(Vorlass) du Literaturarchiv de Marbach, les dossiers

5. - Michael Buselmeier (dir.), Erinnerungen an Wolfgang Hilbig, Heidelberg, Das Wunderhorn, 2008 ; Natascha Wodin, Nachtgeschwister, Roman, München, Antje Kunstmann, 2009 ; Margret Franzlik, Erinnerungen an Wolfgang Hilbig, Berlin, Transit Verlag, 2014. 
des maisons d'éditions est-allemandes situés au Bundesarchiv, ceux du Ministère de la Sécurité d'État du BstU, ainsi que les archives personnelles de proches de Wolfgang Hilbig, en premier lieu de ses compagnes Margret Franzlik, Natascha Wodin et Christiane Rusch, mais aussi la collection privée de Wolfgang Hegewald.

Ces documents s'avèrent une aide précieuse pour reconstituer le projet poétique au fondement de l'œuvre de Hilbig. La correspondance et les journaux intimes ont permis par exemple de retrouver les lectures de l'écrivain, et par conséquent d'éclairer une partie de l'intertextualité qui innerve ses textes, alors même que la bibliothèque de l'auteur, ayant subi plusieurs déménagements, livre trop peu d'informations fiables. Des fragments de sa poétique s'expriment dans le dialogue qu'Hilbig mène dans sa correspondance avec certains de ses contemporains comme les écrivains Franz Fühmann, Wolfgang Hegewald ou Sarah Kirsch. Les projets au long cours restés inachevés et non publiés, comme le projet «Blaue Blume » qui traverse comme souterrainement l'œuvre de Hilbig jusqu'au début des années 1970, suggèrent de voir des cohérences entre les textes qui en dérivent. La durée de maturation d'un texte est particulièrement frappante. Plusieurs décennies peuvent s'écouler, un régime s'effondrer, entre les premières esquisses d'un récit et sa parution.

L'autre pan de la vie de Hilbig que cette biographie documente en détail concerne la confrontation de l'écrivain est-allemand avec le pouvoir. Sa surveillance par la Stasi remonte à 1964, alertée par les propos insolents qu'il tient sur le service militaire qu'il a effectué d'avril à octobre 1963. Dès lors, la vie et l'écriture de Hilbig jusqu'à son départ en RFA en novembre 1985 ne cesseront d'être marquées par les chicanes administratives et politiques. Son courrier est surveillé, ses manuscrits et exemplaires d'auteur confisqués. Non seulement, il n'essuie que des refus de la part des maisons d'édition est-allemandes auxquelles il propose ses textes depuis 1964, mais aucun des livres qu'il publie à l'Ouest, abwesenheit (1979), Unterm Neomond (1982) et Der Brief (1985) n'obtient l'autorisation nécessaire de la part de la RDA. Ils paraissent illégalement. Chaque livre est un combat. Les extraits des rapports de la Stasi, des expertises littéraires des maisons d'édition ainsi que des évaluateurs externes et les nombreux courriers qu'Hilbig adresse au ministère de la culture et aux responsables du SED nous plongent dans les rouages de l'institution littéraire est-allemande. Ils donnent à voir le fonctionnement de la censure, font ressentir la pression qui s'exerce en RDA sur l'individu qui refuse de se plier aux directives officielles, mais aussi l'énergie que déploie le simple ouvrier qu'est Hilbig à l'époque pour défendre, devant les plus hautes instances de l'État et du parti, l'œuvre littéraire dont il se sent investi. Dès lors, les lettres adressées à Klaus Höpcke, ministre adjoint de la culture et responsable de l'administration principale de l'édition et du commerce du livre, ou à l'écrivain Stephan 
Hermlin se lisent comme l'autre œuvre de Hilbig. Elle prend la forme d'une défense de la littérature dans laquelle l'écrivain énonce avec clarté et fermeté sa conception de la littérature et le rôle que devrait jouer l'État. De l'autre côté, les archives du pouvoir dévoilent la perplexité des responsables politiques face à cet individu que ni l'arrestation sous un motif fallacieux au printemps 1978 ni l'amende pour enfreinte à la loi sur les devises en 1979, ni au contraire la parution de ses textes aux éditions est-allemandes Reclam en 1983 (le recueil Stimme Stimme) ne parviennent à faire plier.

\section{Portrait d'un incorruptible}

C'est en somme le portrait d'un incorruptible que dresse sobrement, à travers l'accumulation de matériau, la biographie de Michael Opitz. L'un des fils rouges qui parcourent l'ouvrage est en effet celui de l'éthique de l'écrivain. L'ouvrage met d'abord en évidence certaines permanences du caractère. Ainsi, l'indépendance d'esprit, l'obstination et la force de caractère qui transparaissent chez le jeune Hilbig refusant de se soumettre à l'ordre familial et à la discipline scolaire, se rebellant au service militaire, se retrouvent, mises au service de la littérature, par exemple dans la lecture assidue des romantiques et des classiques de la modernité, situés en-dehors du canon est-allemand. On reconnaît l'éthique de l'écrivain dans son refus de faire allégeance à la RDA lors du discours de remerciement pour le prix Grimm décerné par la ville de Hanau (près de Francfort sur le Main) qu'il prononce en 1983, contrairement à ce que lui avaient demandé les autorités est-allemandes ayant permis son voyage à l'Ouest. Ou encore dans la ténacité avec laquelle, au nom de la responsabilité qu'il porte envers son œuvre, il remue ciel et terre pour faire changer d'avis les différentes instances du pouvoir qui avaient refusé dans un premier temps d'établir le visa d'un an nécessaire pour qu'il puisse bénéficier de la bourse accordée par le fonds littéraire de Darmstadt (en Hesse) en 1985. La figure de l'écrivain intègre surgit à nouveau lorsque Hilbig est mentionné comme l'un des rares à sortir indemne de la querelle littéraire de 1991. La littérature de RDA y était en effet apparue discréditée par les révélations de la collaboration avec la Stasi de nombreux écrivains, y compris ceux de la scène alternative. Hilbig serait dès lors l'un de ceux qui incarnent « l'autre littérature de RDA » (529), dans une expression qui rappelle l'autre Allemagne des écrivains exilés sous le nazisme. Dans ce contexte, il apparaît étonnant qu'Opitz ne fasse pas mention de la correspondance, conservée dans les archives, entre Hilbig et l'un de ces écrivains espions, Rainer Schedlinski ${ }^{6}$.

6. - Stephan Pabst, «'Leere Signifikanz'. Hilbigs Kritik des Poststrukturalismus », in : Frédéric Teinturier et Bénédicte Terrisse (dir.), «Ich» de Wolfgang Hilbig. Littérature, 
Le modèle d'intelligibilité corollaire à la figure de l'incorruptible que mobilise la biographie de Michael Opitz est celui de la rupture. La trajectoire de Hilbig se caractérise en effet par un mouvement de séparation permanent, avec son milieu prolétaire d'origine, avec sa mère, avec ses collègues de sport ou de travail, puis avec sa fille et la mère de sa fille, avec sa ville natale. L'ouvrage consacre ainsi de belles pages au premier recueil de nouvelles de Hilbig qui devait porter le titre " Aufbrüche » (" départs ») avant de s'appeler Unterm Neomond. La rupture se poursuit dans l'Allemagne d'après la chute du Mur où, lors de la remise du prix Lessing à Kamenz en 1997, Hilbig surprend son public en critiquant la manière dont s'est passée l'unité allemande, comparant l'intégration des territoires est-allemands à une annexion colonialiste.

\section{Une autre littérature de RDA?}

Si la figure de l'écrivain intègre et en rupture est une représentation assez commune de Hilbig, la biographie de Michael Opitz place un certain nombre d'accents autrement qui tranchent avec la manière habituelle de représenter la trajectoire d'un écrivain est-allemand. Bien que l'ouvrage documente avec minutie le contexte d'élaboration des textes écrits par Hilbig en RDA livrant ainsi de précieuses informations permettant de comprendre leur réception négative par les autorités culturelles, il ne fait pas de la RDA le prisme majeur d'appréhension de l'œuvre et de la vie de l'auteur. En ouvrant le premier chapitre sur les événements qui se sont déroulés sur le front de l'Est à l'été 1941 et sur les exactions commises par les nazis dans les camps de concentration au moment de la naissance de Hilbig, Opitz place l'écrivain dans le contexte plus général de la littérature allemande au lendemain de l'Holocauste, faisant de la question de la culpabilité et de la mémoire un élément central de l'œuvre de Hilbig. Tout au long de la biographie, il montre comment les allusions à l'Holocauste sont présentes dans toute l'œuvre de Hilbig, de ses premiers poèmes à son dernier roman. Les références à Klemperer, Kertész, Primo Levi et Celan accompagnent discrètement les commentaires des textes, ces classiques de la littérature de l'Holocauste servant en quelque sorte de garants au biographé. Le deuxième chapitre décrit l'enfance de Hilbig comme étant typique de la génération des «enfants de la guerre » (Kriegskinder) ayant grandi sans père et fait des bombardements de Meuselwitz un épisode traumatique et fondateur pour l'écrivain. Opitz prend ainsi acte des derniers travaux sur l'histoire de la littérature est-allemande, que l'on songe à l'ouvrage de Michael Ostheimer ou à celui de Carola Hähnel-Mesnard et Katja Schubert ${ }^{7}$. Il montre aussi comment, dans les derniers textes

identité et faux-semblants, Paris, L'Harmattan, 2013, p. 123-141, ici p. 127 note 8.

7. - Michael Ostheimer, Ungebetene Hinterlassenschaften. Zur literarischen 
de Hilbig, l'horreur que représente l'attaque terroriste du 11 septembre 2001 tend à se superposer à celle de l'Holocauste. Corollairement, Opitz n'interprète pas le passage de l'Est à l'Ouest, la chute du Mur et le changement de régime politique comme une césure dans la vie et l'œuvre de Wolfgang Hilbig. Il n'en fait pas un schème explicatif, ce qui lui permet de ne pas donner dans certaines apories, mais contient aussi le risque de minimiser l'impact de ces faits sur certains textes (Die Kunde von den Bäumen, prosa meiner heimatstraße).

Le déplacement des accents concerne aussi les textes de Hilbig que le biographe choisit de mettre en avant. Ainsi, ce ne sont plus les romans, notamment «Ich» (1993), et le dernier recueil de poèmes Bilder vom Erzählen (2001), qui occupent le premier rang, mais le long poème fragment « prosa meiner heimatstraße » (1988-1992) et le récit Alte Abdeckerei (1991) qu'Opitz qualifie d'opus magnum. Par ailleurs, la biographie n'établit pas de hiérarchie entre textes publiés et textes non publiés, consacrant de nombreuses pages au projet "Blaue Blume ", citant longuement des extraits des histoires de sport écrites par Hilbig dans les années 1960, par exemple «Cross », et abandonnées, « à juste titre », comme il le note (175).

\section{Questions de méthode}

Selon François Dosse, l'alliance entre le travail d'historien, représenté ici par la recherche de sources fiables, et la qualité d'une écriture romanesque caractérise le genre de la biographie. Dans la biographie d'Opitz, la synthèse a particulièrement bien réussi. Dans de nombreux passages, notamment dans les derniers chapitres de la biographie consacrés aux dernières années de la vie de Hilbig, le mélange de document et de récit crée l'« effet de vécu ${ }^{8} »$ tant recherché. Néanmoins, un troisième aspect caractérise spécifiquement la biographie d'intellectuels dont relève le Wolfgang Hilbig de Michael Opitz : la relation entre l'œuvre et la vie. La biographie d'Opitz, comme avant lui celle de Birgit Dahlke, et davantage encore celle de Karen Lohse, tourne sans la saisir autour de cette tache aveugle. Ils ont tous les trois pris le parti d'alterner description d'événements de la vie de l'écrivain et analyse de textes. Opitz n'identifie certes par Hilbig à ses narrateurs ou à ses personnages, mais la juxtaposition des discours (récit de vie et analyse littéraire) à l'intérieur d'une même page crée l'impression d'une forte hétérogénéité qui ne se dissipe que chez le lecteur ou la lectrice acceptant de considérer que l'œuvre et la vie de Hilbig se situent sur le même plan, ne font qu'un. Cette pensée

Imagination über das familiäre Nachleben des Nationalsozialismus, Göttingen, V\&R Uni press, 2013 ; Carola Hähnel-Mesnard et Katja Schubert (dir.), Störfall ? Auschwitz und die ostdeutsche Literatur nach 1989, Berlin, Frank \& Timme, 2016.

8. - F. Dosse, «Préface... », op.cit., p. xv. 
poétique ou mythique est peu compatible avec le travail du biographe, pour qui le rapport entre l'œuvre et la vie constitue le cœur de son sujet. Celui-ci demande à être abordé avec réflexion et méthode. Opitz semble cependant plutôt évacuer le sujet. Après avoir passé en revue les passages des textes de Hilbig qui interrogent la notion de biographie sans les analyser réellement, l'introduction se borne à rappeler que chez Hilbig la vie est subordonnée à l'écriture et que personnages et narrateurs ressemblent tant à leur auteur qu'il est justifié de parler de textes autofictionnels, avant d'ajouter que Hilbig utilise sa propre vie comme matériau et que la plupart des critiques littéraires lisent ses textes de manière autobiographique (15). Si, contrairement à Karen Lohse, Opitz ne fait pas de l'œuvre de Hilbig son matériau biographique, le statut des textes littéraires et de leur commentaire, qui relève parfois de la simple paraphrase, reste trop flou. Comme dans les précédentes biographies, les textes de Hilbig semblent convoqués pour confirmer la biographie, dans une logique associative propre à la subjectivité du biographe. Le fait que l'ouvrage ne contient qu'un index des noms propres et aucun index des œuvres de Hilbig citées, et que, dans cet index des noms propres, figurent en italique, au milieu des personnes réelles, les noms des personnages des récits de Hilbig en dit long sur le flottement méthodologique qui préside à l'ouvrage. Les limites éthiques de la transgression des frontières entre l'œuvre et la vie dans le genre factuel qu'est la biographie apparaissent ainsi dans les premiers chapitres de l'ouvrage consacrés à l'enfance et à l'environnement familial de l'écrivain. L'empathie avec l'objet de la biographie, caractéristique d'une telle entreprise, conjuguée au défaut de méthode qui fait prendre certains fragments de prose retrouvés dans les archives et le roman de Natascha Wodin Nachtgeschwister pour des documents véridiques, transforme ces chapitres en véritable procès contre la mère. Il est probable que cette interprétation de Marianne Hilbig soit en réalité fondée, de manière plus ou moins consciente, sur le récit Die Weiber (1987), récit jubilatoire et excessif qui joue notamment avec les catégories de la psychanalyse, autre tache aveugle de la biographie de Michael Opitz.

Dans un article sur les biographies du poète expressionniste Georg Trakl, Philippe Payen de la Garanderie distinguait ce qu'il appelait le «modèle de la biographie unitaire », dans lequel l'œuvre et la vie ne font qu'un, où le biographe s'appuie sur l'œuvre pour étayer la biographie et le poète est identifié à ses personnages (232-233), ayant cours dans les années 1920, la « biographie dédoublée », « renonçant à toute implication du biographique dans le poétique » (234), que l'on retrouve dans les années 1950, et la « biographie paradoxale » des années 1990, qui 
problématise la «faille » entre l'œuvre et la vie ${ }^{9}$. L'analyse passionnante que fait Opitz du rapport à Strindberg dans le dernier roman de Hilbig, Das Provisorium (2000), à partir d'une citation sur la teneur autobiographique de l'œuvre, transforme l'enquête sur la vie en enquête sur le texte. Ce passage ouvre une brèche vers ce qui pourrait ressembler à une «biographie paradoxale » de Hilbig, intégrant à son écriture la réflexion sur le rapport entre l'œuvre et la vie qui permettrait de dépasser le stade de la «biographie unitaire».

Le traitement du matériau exhumé des archives n'échappe pas, lui non plus, au défaut de méthode. La biographie de Michael Opitz a certes le mérite de donner à lire des textes inconnus, mais elle occulte la question de la genèse de l'œuvre qui montrerait à l'aide d'un dossier génétique les liens entre un texte et ses avants-textes, rendrait ces derniers productifs pour l'interprétation du geste créatif hilbigien, interrogerait les frontières de l'œuvre. Les textes exhumés apparaissent au contraire trop souvent comme de simples objets à admirer, dénués de toute valeur heuristique. Quelques confusions entre textes non publiés et textes publiés, partagées pour certaines avec les Hilbig-Werke ${ }^{10}$, semblent trahir la fascination pour son matériau archivistique à laquelle Opitz cède parfois.

\section{Le biographe en chiffonnier}

On pourrait se demander si ce ne sont pas finalement les archives qui sont aux commandes de l'ouvrage et si le principe qui guide l'écriture de cette biographie n'est pas simplement d'exhumer le plus grand nombre de matériau inconnu possible, au point de faire apparaitre ce que l'on connaît de Hilbig comme la partie émergée de l'iceberg, de modifier les équilibres et les proportions, de déplacer les centres de l'attention habituels. Ainsi, les trois cents premières pages de l'ouvrage couvrent la période précédant les premières publications de Hilbig, mettant au jour des textes non publiés ou jugés trop méconnus. En se faisant chiffonnier de l'œuvre de Hilbig, sur le modèle de cette figure benjaminienne qu'il

9. - Philippe Payen de la Garanderie, « Georg Trakl (1887-1914) : le paradoxe biographique », in : ibid., p. 229-242, ici p. 232-235. Les trois biographies étudiées sont celles d'Erwin Mahrholdt (1925), Wolfgang Schneditz (1951) et Hans Weichselbaum (1994).

10. - Le texte «Eine Antwort» dont Opitz cite la source dans les archives (p. 26) a été publié en 2002 : Wolfgang Hilbig, «Lieber Lord Chandos (1). Aufruf zum Widerstand. Warum wir dem Zerfall trotzen müssen », Frankfurter Allgemeine Zeitung, 19.07. 2002, $\mathrm{n}^{\circ} 165$, p. 37. C'est également le cas de la cinquième partie du long poème « prosa meiner heimatstraße » que Opitz (p. 211) et le $1^{\text {er }}$ volume des Hilbig-Werke indiquent à tort comme inédit (p. 502) : Wolfgang Hilbig, « prosa der heimatstraße (Fragment) 5. vaterland der asche » in : Thomas Rietzschel (Hrsg.), Über Deutschland. Schriftsteller geben Auskunft, Leipzig, Reclam, 1993, p. 116-119. 
met en lumière dans Die Kunde von den Bäumen, Opitz semble vouloir sauver précocement de l'oubli un matériau brut qu'il reste à façonner. Malgré sa méthodologie brouillonne - grief qui est un poncif de la critique du genre biographique ${ }^{11}$, l'ouvrage de Michael Opitz est d'ores et déjà une référence. Par le foisonnement de son matériau, la clarté de ses explications et la richesse de ses analyses, il parvient à donner corps à l'écrivain Hilbig sans tomber dans le mythe, ni l'hagiographie. Cette somme est aussi une incroyable mine d'idées pour de futurs travaux sur l'écrivain.

Bénédicte Terrisse 
Norbert Waszek (Hrsg.), Rosenzweigs Bibliothek. Der Katalog des Jahres 1939 mit einem Bericht über den derzeitigen Zustand in der tunesischen Nationalbibliothek, Freiburg/München, Verlag Karl Alber, 2017, $156 \mathrm{~S}$.

Décédé en décembre 1929, alors qu'il allait avoir 43 ans, Franz Rosenzweig laissait derrière lui une œuvre philosophique et pédagogique au caractère novateur, que l'on songe à L'Étoile de la Rédemption, son écrit majeur paru en 1921, magistralement commenté par Stéphane Mosès (Système et Révélation, 2e éd. Bayard, 2003), ou au Freies Jüdisches Lehrhaus de Francfort, espace d'écoute, de dialogue et de questionnement à l'infini, prônant un judaïsme vivant, fondé à l'été 1920. Atteint de paralysie en 1922, il n'en poursuivit pas moins la traduction de la Bible entamée avec Buber, qui paraîtra dans son intégralité en 1962. Bien que cette disposition ne fût pas clairement formulée dans son testament, il léguait également à son fils unique, Rafael Nehemia, une bibliothèque dont le catalogue révèle plus de 2600 entrées et dont Norbert Waszek retrace dans son introduction l'histoire mouvementée qui, dit-il, « pourrait fournir matière à un roman ». Rafael ayant quitté l'Allemagne pour faire son alya au lendemain du pogrome des 9 et 10 novembre 1938 , la bibliothèque fut embarquée en direction de la Palestine en 1939 sur un cargo allemand arraisonné par la flotte britannique à l'approche de la Tunisie, à l'époque sous protectorat français, et déposée à la Bibliothèque de Tunis. Au lendemain de la guerre, n'ayant ni les moyens matériels et financiers, ni sans doute le désir de renouer avec le passé et la culture d'une Europe qui avait laissé massacrer ses Juifs, Rafael vendit le fonds à la Bibliothèque de Tunis - ultime rébellion peut-être, aussi, contre l'imposante figure d'un père dont il ne partageait pas les réserves à l'égard du sionisme.

Paru une première fois dans le Rosenzweig Jahrbuch (Bd.8/9, 2014/2015), le catalogue fut établi en 1939 à la demande des autorités allemandes, accompagné de l'attestation que les ouvrages avaient été acquis au nom de Rafael avant l'installation au pouvoir de Hitler (Rafael avait alors onze ans !), condition sine qua non pour le transfert de ces biens « juifs » hors de l'Allemagne nazie. «Ruse de la raison? », se demande Norbert Waszek, ce qui n'était qu'une « chicane » parmi tant d'autres devait se transformer en "bénédiction » pour les chercheurs qui peuvent ainsi entrevoir « l'atelier » de Rosenzweig. Destinée à un public plus vaste, cette seconde édition est le fruit d'une collaboration entre Norbert Waszek, qui en a assuré la direction, Wolfgang Fink, qui s'est rendu à Tunis afin de constater l'état de la bibliothèque et de vérifier son adéquation avec la liste des ouvrages figurant au catalogue, et Bernd Füllner dont quatre étudiantes ont travaillé à la numérotation, la correction et l'aération des entrées. 
Sur un plan historique et sociologique, la bibliothèque offre un aperçu de l'environnement et des choix culturels de la bourgeoisie juive assimilée férue de culture allemande, d'art et de musique, d'une intelligentsia plus proche du judaïsme libéral que de l'orthodoxie, peu acquise aux idées du marxisme d'une part et du sionisme d'autre part (on note l'absence notoire des écrits de Marx et de Herzl, ce qui laisse supposer que Rosenzweig ne voyait pas en eux des bâtisseurs d'avenir, car il ne faut pas oublier que ce fonds était destiné à sa postérité). Les " classiques » de la littérature européenne (les auteurs contemporains, représentants de la modernité, occupent relativement peu de place) côtoient les œuvres de Lessing et de Schiller et, au tout premier rang, de Goethe. L'idéalisme allemand (Hegel en tête, auquel Rosenzweig consacra sa thèse, Schelling et Fichte) et l'histoire de la Prusse (Bismarck en particulier) et de la genèse et de la montée du nationalisme allemand apparaissent également en bonne place, ce dont il ne faudrait pas hâtivement conclure que Rosenzweig se serait rallié à l'esprit d'un Ranke ou d'un Treitschke. Plusieurs entrées témoignent d'ailleurs de l'attention portée au phénomène de l'antisémitisme.

Plus intéressant est, si l'on considère la bibliothèque dans son ensemble, ce qu'elle nous apprend de la position occupée au sein du judaïsme allemand par celui qui rêvait de créer en Allemagne un foyer du judaïsme (le Freies Jüdisches Lehrhaus) et considérait L'Étoile de la Rédemption comme un cadeau « fait à l'esprit allemand par son enclave juive » (lettre à Rudolf Hallo datée de fin janvier 1923). Si le catalogue en soi ne reflète pas le « retournement » effectué par celui que Lévinas qualifiera d'« hégélien repenti », il illustre cette « liberté du "et" » revendiquée par Rosenzweig dans une lettre à Hélène Sommer datée du 18 janvier 1918, son choix d'emprunter une troisième voie, en marge de l'assimilation et du sionisme, celle de la "dissimilation » (terme qu'il utilise dans son journal à la date du 16 janvier 1928) dans laquelle il voyait le chemin menant vers l'avenir de la diaspora. D'où un nombre presque égal d'entrées relatives aux sources juives (aux livres bibliques et à l'hébreu biblique en vue entre autres de la traduction entreprise avec Buber, à Jehuda Halevi dont Rosenzweig traduisit les poèmes, au Baal Shem Tov, aux écrits de Leopold Zunz et de Heinrich Graetz, etc.) et au judaïsme contemporain en pleine effervescence (cf. les entrées relatives aux écrits de Hermann Cohen et des collaborateurs de Rosenzweig au Lehrhaus dont nous ne citerons ici que Buber, Ernst Simon et les publications à teneur plus « locale » de Rudolf Hallo). Féru de théologie et d'histoire des religions et passionné par l'étude des langues (double intérêt que reflète le catalogue), Rosenzweig fut également l'un des précurseurs du dialogue judéo-chrétien, même si paradoxalement, la plupart de ses interlocuteurs étaient des proches, juifs convertis : Eugen Rosenstock-Huessy et ses cousins Hans et Rudolf Ehrenberg (le cata- 
logue fournit une ample bibliographie de leurs écrits pouvant alimenter les recherches autour de la «nouvelle pensée » que Rosenzweig entendait promouvoir avec ses amis).

Une attention particulière doit enfin être portée à la reproduction en fac-similé d'extraits de la Phénoménologie de l'Esprit de Hegel et du Système de la philosophie transcendantale de Schelling. Ces quelques pages, soigneusement sélectionnées par Myriam Bienenstock, portent des annotations de la main de Rosenzweig et constituent, en marge du catalogue, une invitation et une réelle incitation à la recherche, informant sur la lecture que faisait Rosenzweig de ces passages et laissant percevoir, au bas d'une page, l'amorce d'une prise de distance.

Pour une exploitation maximale du fonds de cette bibliothèque, on pourrait bien sûr imaginer son retour en Allemagne ou son transfert aux USA. Ou, ajouterions-nous, on pourrait rêver qu'elle poursuive son voyage entamé en 1939, en direction aujourd'hui de la prestigieuse Université Hébraïque de Jérusalem et du Franz Rosenzweig Minerva Research Center. Partant du chemin tracé par Myriam Bienenstock, Norbert Waszek envisage quant à lui un autre avenir, qu'il soumet à la discussion, consistant en une numérisation et une mise en ligne sur internet des ouvrages (ou de certains ouvrages ou extraits) avec les annotations de Rosenzweig, celles-ci devenant dès lors accessibles à tous les chercheurs. Cette solution qui rendrait pleinement hommage à la Bibliothèque Nationale de Tunis, détentrice et conservatrice du fonds, pourrait être soutenue financièrement par des institutions de recherche et des fondations. C'est cette suggestion que nous voudrions ici relayer afin que vive cet héritage de l'un des plus éminents penseurs du siècle dernier.

[Pour les références à la correspondance et au journal de Rosenzweig, cf. Briefe und Tagebücher, 2 Bände, hrsg. von Rachel Rosenzweig und Edith Rosenzweig-Scheinmann unter Mitwirkung von Bernhard Casper, Haag, Nijhoff, 1979 ; pour le texte d'Emmanuel Lévinas consacré à Rosenzweig, voir Hors sujet, Paris, Le Livre de Poche (biblio essais), 1997, p. 67-84].

Andrée Lerousseau

Dirk Weissmann, Métamorphoses interculturelles. Les Voix de Marrakech d'Elias Canetti, Paris, Éditions Orizons, 2016, 338 p.

L'ouvrage de Dirk Weissmann est l'un des premiers ouvrages en français entièrement consacré aux Voix de Marrakech et doit être salué en tant que tel. Il se donne pour objectif d'étudier précisément l'intégralité de l'œuvre afin d'en mettre au jour les présupposés mais aussi les conséquences, et l'on peut considérer comme une gageure de proposer un ouvrage de 338 pages, ce dont D. Weissman est bien conscient (p. 301), 
pour un opuscule original d'à peine une centaine, mais la richesse des Voix de Marrakech justifie, toujours selon D. Weissmann, l'ampleur «d'une analyse aussi exhaustive que possible du texte » (p. 17).

On saluera une très grande connaissance de l'œuvre générale de Canetti ainsi qu'une explication précise du contexte particulier à l'intérieur du projet canettien. Les notions capitales - la lutte contre la mort, la défense de l'écrivain comme gardien des métamorphoses, la théorie du masque acoustique et les origines multiculturelles de l'auteur - ainsi que les étapes décisives de sa vie - notamment son enfance « comme matrice de la polyphonie identitaire qui est la sienne » (p. 169) - sont également bienvenues pour contextualiser les conditions d'émergence ainsi que l'horizon d'attente de l'ouvrage. Les données historiques et politiques nécessaires afin de comprendre la situation du pays, le Maroc avec les divisions ethniques entre Berbères et Arabes, et de la ville, Marrakech, à un moment particulièrement important de leur histoire, à une période pivot pour l'évolution du pays, permettent de mieux saisir le caractère particulier des Voix de Marrakech.

Le livre est construit en cinq parties : la première fait office d'introduction générale à l'ouvrage et à l'auteur ainsi que d'annonce de la problématique générale qui vise à faire de Canetti un auteur s'interrogeant sur l'identité, mais aussi la mondialisation et le (post)colonialisme. Les parties deux, trois et quatre correspondent à la structuration de l'ouvrage en trois sections que dégage $\mathrm{D}$. Weissmann : les chapitres un à six (partie introductive générale sur l'identité islamique de la ville) et neuf à quatorze (préférence topographique de la place Jemâa El Fna) entourent le cœur du travail consacré au judaïsme à Marrakech par la découverte du Mellah, le quartier juif (p. 50) et sont tous reliés par un réseau thématique protéiforme ainsi qu'une série d'oppositions structurantes (locales, sociales, socio-politiques, genrées, anthropologiques, physiques, normatives, religieuses, sonores et émotionnelles, pour ne citer que les plus fréquentes, p. 51). Enfin la cinquième partie constitue à la fois une conclusion, un rappel des différentes problématiques et une tentative d'élargissement de la perspective.

Dans la première partie, D. Weissmann rappelle la biographie et le parcours intellectuel d'Elias Canetti, " l'écrivain par excellence du pluriculturalisme et du plurilinguisme européens » (p. 24) qui conjugue " l'éloge de la diversité culturelle avec la pensée universaliste des Lumières » (p. 29-30) impliquant «plasticité et dynamisme » (p. 32). Il réinscrit l'ouvrage dans un cadre générique (récit de voyage, document, autobiographie, autofiction par exemple) et dans l'évolution de Canetti (retour sur sa judéité, découverte du monde arabe) tout en relevant la posture particulière prise par Canetti avec cet opuscule « écrit après un voyage » et qui serait comme une «illustration littéraire des théories exposées dans Masse et puissance, publié en 1960 »(p. 42), autrement 
dit six ans après le voyage effectué à Marrakech dont le texte ne sera publié qu'en 1968. D. Weissmann aimerait pouvoir considérer les Voix de Marrakech comme l'exemple même de la métamorphose canettienne, envisagée comme «métamorphose interculturelle » (p. 61), en insistant sur les processus de perception, qu'il s'agisse de l'ouïe ou de la voix.

Dans la partie centrale (2 à 4), D. Weismann analyse chapitre par chapitre l'ouvrage en question qui s'ouvre par une sorte de cadre narratif, par exemple par la couleur rouge de la ville qui fait écho au symbolisme de cette couleur dans l'histoire d'une vie de l'auteur, notamment $L a$ Langue sauvée, ainsi que sur l'animal, en l'occurrence le chameau, «à la fois comme limite et comme matrice de l'humain» (p. 70). D'autre part cet incipit est aussi à lire comme le désir « d'inscrire le récit du voyage à Marrakech dans le cadre d'une mémoire des catastrophes du $\mathrm{XX}^{\mathrm{e}}$ siècle » (p. 82) pour faire de la ville un lieu de mémoire, en particulier de « l'identité juive perdue de Marrakech» (p. 215). Ce qui importe à Canetti, c'est « l'exemplarité des quelques phénomènes qu'il observe et interprète, et non pas l'objectivité ou l'exhaustivité de la description du lieu visité » (p. 87). L'ensemble de l'ouvrage pourrait se lire comme une sorte de critique de la modernité occidentale, voire anticapitaliste, si Canetti ne venait tempérer ses propres jugements par une relativisation de la situation marocaine : qu'il s'agisse de vanter les mérites de la communication que l'institution culturelle des souks permet ou qu'il adresse une critique envers l'admiration sans borne d'une grande partie de la communauté juive marocaine pour le modèle de vie nord-américain. Feignant de ne rien connaître à la langue et à la culture marocaine, Canetti se place dans la situation de l'observateur objectif - il semble que le terme de « voyeur » serait plus approprié -, même s'il se trahit à plusieurs reprises lorsqu'il doit reconnaître s'être renseigné antérieurement à son voyage. C'est ce manque « d'honnêteté » vis-à-vis du lecteur qui rend la lecture de l'ouvrage quelque peu difficile, car le lecteur ne sait pas à quel narrateur il a à faire ni quels sont les raisons de la manipulation opérée : peut-être $\mathrm{D}$. Weismann aurait-il pu tenter de donner un début de réponse à cette question de première importance étant donné le point de vue éthique que Canetti prétend régulièrement adopter, notamment en ce qui concerne le rapport aux femmes. Les Voix de Marrakech rappellent la «valeur collective » de l'oralité et la «fonction éminemment sociale attribuée à l'écriture » (p. 234). Le chapitre le plus abouti et le plus réussi est certainement celui consacré à "Shéhérazade »l'avant-dernier du recueil de Canetti - dans lequel $\mathrm{D}$. Weismann révèle son talent d'observateur du détail conjugué à une hauteur de vue qui replace l'ensemble dans un contexte littéraire et politique éclairant.

La troisième partie replace l'ouvrage dans une réflexion sur les difficiles rapports entre Occident et Orient, en 1954 déjà mais plus encore aujourd'hui, et sur leur complexité, notamment autour de la question 
de l'orientalisme qui a suscité controverse et lectures divergentes qui vont « jusqu'à s'opposer diamétralement » (p. 307) : « La complexité identitaire de l'auteur, la dimension transnationale, transculturelle et translingue de son parcours mettent à mal le schématisme et l'essentialisme inhérents à certains textes fondateurs des études postcoloniales » (p. 310). D. Weismann en arrive également à la conclusion que la métamorphose est un « principe littéraire plus qu['un] concept scientifique » (p. 317).

On soulignera une finesse d'analyse presque toujours pertinente, qui cependant a quelquefois tendance, peut-être, à une surinterprétation, voire à livrer toutes les interprétations possibles sans prendre une position personnelle (p. 96 et 102-103 par exemple), ce qui est peut-être dû, dans une sorte d'angélisme postcolonial, à une trop grande empathie et confiance en l'auteur. La méthode adoptée est celle d'une étude immanente (close reading, p. 17), qui suit le texte chapitre par chapitre, et se justifie certes, mais a le défaut de ses qualités dont l'inconvénient majeur est d'entraîner une série de redites et de répétitions parasitaires. Le style est fluide, alerte, le texte est rédigé dans un français impeccable, malgré quelques coquilles amusantes : ainsi p. 14 Auto-da-Fée (!) pour le titre de l'unique roman de Canetti ou bien p. 16 note $4:$ « la première monographie est parue en $2011 »$ suivie de l'indication de l'ouvrage en question dont la date de publication est 2009 ! ou p. 200 « en l'exil en août 1935 ». On pourra regretter que l'auteur de l'étude soit sujet à une empathie peut-être trop grande avec Canetti, en voulant à toutes fins voir en lui un personnage novateur par ses interrogations de type postcolonial et qui semble lui interdire une prise de distance critique suffisante vis-à-vis d'Elias Canetti, même s'il reconnaît l'ambiguïté et la complexité de l'auteur et de l'homme.

Il est regrettable peut-être que les concepts de liminalité et d'hybridité restent quelque peu sous exploités, même si la conclusion (p. 319) tente de les situer dans le contexte général de l'œuvre canettienne. Mais le plus important réside dans la remarque finale selon laquelle Canetti rendrait possible une analyse de la littérature en « termes de trajectoire plus que d'aires culturelles, qui déplace les frontières au lieu de les durcir, qui envisage des relations et communications davantage que des territoires fixes » (p. 323), et constituerait donc un plaidoyer pour une littérature en mouvement, une idée tout à fait passionnante que D. Weismann, nous l'espérons, développera dans ses ouvrages à venir. 
Sonia Combe, Antoine Spire, Maladie et privation d'amour. De Christa Wolf à Canguilhem, pour un retour à la clinique, Lormont, Le bord de l'eau, 2017, $156 \mathrm{p}$.

En novembre 1984, Christa Wolf fut invitée à Magdebourg afin de prononcer une conférence lors du premier congrès du groupe de travail sur la «gynécologie psychosomatique » qui venait de se former en RDA.

Dans « Krankheit und Liebesentzug. Fragen an die psychosomatische Medizin » (« Maladie et privation d'amour. Questions à la médecine psychosomatique »), texte publié ensuite dans le recueil d'essais Die Dimension des Autors (Berlin, Aufbau, 1986), l'auteure est-allemande se réjouit de la naissance de ce groupe de travail en RDA qui remet en question ce qu'elle appelle la " médecine scientifique ». Convaincue, en tant que personne, de la nécessité de tenir compte de la "psyché » dans le processus thérapeutique, consciente que les progrès techniques éloignent de plus en plus le patient des médecins qui, eux, ont tendance à considérer le corps comme une machine, Wolf appuie son argumentation sur de nombreux témoignages de femmes. Lauteure aborde ensuite des thématiques qui lui sont chères et qui traversent également son œuvre littéraire. Elle interroge les conséquences de la disparition du matriarcat sur la condition féminine, mène une réflexion sur la déconsidération des femmes à travers les siècles (la société leur déniant à chaque fois les valeurs les plus importantes de l'époque, que ce soit la foi au Moyen Âge, ou l'intellect, plus tard), tout en se référant aussi bien à l'histoire littéraire ou encore à l'actualité littéraire en RDA, lui permettant au passage de pointer le décalage entre discours officiel sur la question des femmes et réalité vécue.

Ce texte de Christa Wolf témoigne de l'intérêt que celle-ci a toujours porté aux questions de société, et en le lisant, on s'aperçoit qu'il n'a rien perdu de son actualité. C'est ce qui a conduit Sonia Combe à republier cette conférence aujourd'hui, et à la faire entrer en résonance avec le philosophe français Georges Canguilhem introduit par Antoine Spire. La petite publication est donc le fruit d'un travail à quatre mains combinant travail historiographique et de contextualisation mené par Sonia Combe pour le texte de Christa Wolf avec une redécouverte de certains aspects de la pensée canguilhemienne sur la maladie et la clinique, et une observation lucide de l'univers hospitalier d'aujourd'hui, sous la plume avisée d'Antoine Spire.

Publiée une première fois en français par Sonia Combe dans Les Temps modernes en avril 1989, la conférence de Christa Wolf paraît aujourd'hui dans une traduction revue, et surtout accompagnée d'importantes informations sur sa genèse. En effet, l'historienne s'est rendue dans les archives de l'Akademie der Künste à Berlin où elle a pu consulter la correspondance entre l'écrivaine est-allemande et le docteur Paul 
Franke qui, en février 1984, l'invita à venir prononcer une conférence, considérant que « [t]ous vos livres, et en particulier celui que je viens de lire, Cassandre, montrent que c'est vous qui êtes compétente pour parler de "la femme" » (p. 21). Christa Wolf accepte sans hésitation, disant qu'elle s'intéresse depuis longtemps à la «médecine psychosomatique », mais lorsque le docteur Franke lui fait part des difficultés rencontrées par son groupe de travail face à la médecine officielle, Wolf lui propose de se retirer du projet, pensant que sa présence pourrait « faire courir des risques à votre association » (p. 26). En effet, comme l'explique Sonia Combe, Christa Wolf était tombée en disgrâce à cette époque et les autorités est-allemandes voulaient d'abord empêcher sa présence au congrès, ce qu'ils n'ont finalement pas fait.

En dehors de ces extraits inédits de la correspondance avec le docteur Franke, l'historienne nous propose deux textes qui permettent de contextualiser la conférence de Wolf. Ainsi, un premier texte revient sur la question des femmes en lien avec le "patriarcat socialiste "réel" ", pointant le décalage entre nombre de lois progressistes en faveur de l'égalité entre hommes et femmes que les historiens voient aujourd'hui encore comme un avantage du système socialiste (égalité des salaires, possibilités de faire des études, prise en charge des enfants, droit à l'avortement, etc.) et le maintien d'une mentalité patriarcale conduisant objectivement à la persistance des inégalités, du sexisme et du machisme. En effet, l'égalité des droits ne signifiait pas l'émancipation attendue. Dans ce contexte, Sonia Combe souligne le rôle joué par la littérature de femmes de RDA qui a régulièrement mise en lumière les contradictions du régime, mais elle met également en évidence l'écart, voire l'incompatibilité de cette démarche avec le féminisme occidental. Dans un deuxième texte, l'historienne plaide pour une historiographie qui tienne compte d'une « approche par le genre » et de la biopolitique, afin de pouvoir mesurer plus précisément le rapport entre le pouvoir, représenté par l'État et ses institutions, et la société, ainsi que les marges de manœuvre que pouvait y avoir l'individu. Privilégiant les apports de l'histoire sociale face à la seule étude des mécanismes de domination et de répression, Sonia Combe élargit ici le regard au-delà de la RDA, en rendant compte des débats actuels sur les femmes comme acteurs sociaux (la « women's agency ») dans d'autres pays de l'ancien bloc de l'Est, et notamment en Bulgarie. La question de savoir si les régimes communistes ont fait faillite en matière d'émancipation de la femme ou non, reste donc ouverte jusqu'à aujourd'hui.

Dans la dernière partie du livre, Antoine Spire revient sur la conférence de Christa Wolf et son rapport à la médecine psychosomatique, en créant des liens avec la pensée philosophique de Georges Canguilhem. Ce dernier s'est toujours révolté contre la déshumanisation de la médecine moderne occidentale, essayant de réhabiliter l'individualité du 
malade, son ressenti et sa souffrance, et de souligner l'importance de l'échange langagier avec le patient. Antoine Spire analyse ensuite en connaisseur - il a été directeur du Département Recherche en SHS de l'Institut national du cancer - les défauts d'un système médical et hospitalier hautement normé qui éloigne de plus en plus les médecins de la personnalité et du corps des patients. C'est là que l'actualité rejoint la situation décrite par Christa Wolf au milieu des années 1980. Visiblement, la médecine psychosomatique a du mal à se faire une place dans l'univers médical.

Carola Hähnel-Mesnard

Siegfried Kracauer, Politique au jour le jour 1930-1933, traduit de l'allemand par Jean Quétier, avec la collaboration de Katrin Heydenreich - préface de Jean Quétier, Paris, Éditions de la Maison des Sciences de l'Homme, 2017, 184 p.

La Collection «Bibliothèque allemande » des Éditions de la Maison des Sciences de l'Homme propose, dans sa série Philia dédiée à l'histoire des idées, un choix de cinquante Feuilletons publiés par Siegfried Kracauer entre le 3 mai 1930, quelques semaines après l'entrée en fonction du cabinet minoritaire dirigé par Heinrich Brüning, et le 2 mars 1933, jour où le sociologue, fuyant Berlin et l'incendie du Reichstag qu'il aborde dans ce dernier texte, arrive à Paris. Ces textes, savamment introduits et traduits avec beaucoup d'acuité, sont très intelligemment accompagnés d'un appareil critique issu de l'édition allemande des œuvres complètes de Kracauer et enrichi par des ajouts judicieux du traducteur pour aider son lecteur francophone. Ils dévoilent d'une part toute la richesse des pages culturelles de la Frankfurter Zeitung, grand quotidien démocrate dont Kracauer est le correspondant berlinois entre 1930 et 1933. De nombreux thèmes de la vie quotidienne sont ainsi couverts, le lecteur assiste à des audiences de tribunaux, à des conférences universitaires, aux départs dominicaux des citadins à la campagne « pour se remettre de la ville » (6), il prend le train BerlinHambourg, le « Hambourgeois volant» (168); il se rend au Lunapark, parc d'attractions construit en 1904 et rénové en 1930, que Kracauer juge avec distance :

La foule quitte en bon ordre ce lieu de plaisir qu'elle a parcouru en bon ordre. Elle s'est évadée pour un court moment du quotidien organisé pour se jeter dans un bonheur organisé lui aussi selon des plans rationnels. L'illusion est tout (10).

Le lecteur entre dans les grands magasins où Noël commence dès la mi-novembre, dans des cafés à la mode, dans des cabarets ou des variétés. 
Les textes montrent aussi toute la tension politique de ces dernières années où, sur le plan politique, la République de Weimar se transforme en démocratie présidentielle (cabinet Brüning) puis en dictature présidentielle (cabinet von Papen, cabinet Schleicher) avant qu'Hitler ne soit appelé au pouvoir et nommé chancelier par le président Hindenburg le 30 janvier 1933. Les Feuilletons disent la pauvreté, la crise économique, les tensions politiques, la montée de l'antisémitisme, une sorte d'irrésistible avancée de Weimar vers l'issue fatale. Le 1er mai 1930 se déroule en deux temps, le cortège de la manifestation communiste devance celui de la social-démocratie, " on veille à ce que les cortèges ennemis ne se retrouvent pas au même moment au même endroit » (3). Kracauer décrit la Leipziger Strasse, grande artère berlinoise qui panse ses plaies après un défilé nazi en octobre 1930 : le sol est jonché de verre, «les jets de pierre semblent avoir visé une religion, car pour l'essentiel ce sont les noms juifs qui sont touchés » (28). Il évoque l'apparition «sans bruit » (114) du drapeau à croix gammée dans les rues de Berlin lors de l'élection à la diète régionale de Prusse le 24 avril 1932, et l'oppose au drapeau noir, rouge et or de la République, drapeau qui « donne l'impression, d'un seul point de vue optique, d'une honorable dame plongée dans une rude société » (114). Kracauer évoque aussi la «belle et digne cérémonie » (127) à la mémoire de Walther Rathenau, ministre des Affaires étrangères du Reich assassiné en 1922 par des membres de l'Organisation Consul, organisation secrète d'extrême droite née après la dissolution des corps francs :

Pour nous qui l'avons vécue, cette époque semble déjà presque relever de l'histoire. Pourtant, quand on a hissé le drapeau de la république, elle s'est mise à remonter en même temps, et elle est redevenue présente à nos yeux en ces jours tout aussi troubles (126).

Le livre se clôt sur l'évocation de Berlin au lendemain de l'incendie du Reichstag, "ses parois de verre ont brûlé pour la plupart, et des trous noirs bâillent un peu partout entre les côtes de ce squelette clair » (182). Dans la foule qui tourne autour de l'édifice, Kracauer relève les « employés, bourgeois, ouvriers, chômeurs, soldats de l'armée du Reich » (182) - et les écoliers :

Sans arrêt, de nouveaux groupes d'écoliers se mêlent aux adultes. Ils flairent l'agitation et se réjouissent de l'événement sans en prendre la mesure. Quand ils seront grands, ils apprendront dans les livres d'histoire quelle était réellement la signification de l'incendie du Reichstag (183).

On l'aura compris : cette publication est la bienvenue pour le public francophone, à la fois pour lui faire connaître une autre facette de 
Siegfried Kracauer et pour lui faire découvrir un riche tableau d'une République de Weimar en train d'agoniser.

Martine Benoit

Heinrich Mann, Le Roman d'Henri IV, t. 1, La Jeunesse du roi (612 p.); t. 2, Le Temps de l'accomplissement (789 p.), traduit de l'allemand par Albert Kohn, postfaces de Hans Hartje, Paris, Éditions Petra, 2015.

Hans Hartje, maître de conférences en littérature comparée à l'Université de Pau et des Pays de l'Adour, a eu la belle idée de travailler à une réédition du roman qu'Heinrich Mann écrivit pendant ses années d'exil en France (la première partie parut en 1935, la seconde en 1938) et qu'il qualifia lui-même de "roman français de langue allemande » (lettre à Félix Bertaux datée du 7 octobre 1934). Reprenant la traduction d'Albert Kohn parue en 1972 aux Éditions Gallimard et épuisée, Hans Hartje accompagne cette réédition du texte que Lionel Richard écrivit en 1972 pour le Magazine littéraire et dans lequel celui-ci rappelait que Le Roman d'Henri IV était « un acte politique : Heinrich Mann $\mathrm{y}$ exprime les analogies de la fin du seizième siècle avec l'Europe des années trente. Une Europe sous la menace fasciste, et dans laquelle le roi Henri peut apparaître comme l'antithèse de Hitler » (T.1, p.602). Heinrich Mann lui-même écrivait dans un article paru en 1939:

Chaque fois que nous abordons un personnage historique, nous le rattachons aussi à notre époque. Sinon, nous ferions tout au plus un beau portrait, mais qui nous resterait étranger. Non, le personnage historique, dont nous nous emparons deviendra, que nous le voulions ou non, un exemple forgé à partir de ce que nous avons vécu, il ne va pas seulement signifier, il sera ce que notre époque produit ou ce dont malheureusement elle est privée. Nous le montrerons avec douleur à nos contemporains auxquels nous dirons : voyez donc cet exemple.

Dans une postface de quelque 25 pages, Hans Hartje expose le travail d'Heinrich Mann, son engagement antifasciste, ses échanges épistolaires avec Félix Bertaux, l'enthousiasme de Thomas Mann à la lecture du roman (« une œuvre qui fait honneur à la maison d'édition des émigrés et à l'émigration dans sa totalité et qui, dès lors que les choses auront changé, sera portée aux nues en Allemagne »- note Thomas Mann dans son Journal le 25 septembre 1935). On profitera en outre de cette réédition pour relire les livres de Chantal Simonin (Heinrich Mann et la France. Une biographie intellectuelle, Presses Universitaires du Septentrion, Villeneuve d'Ascq, 2005) et d'André Banuls (Heinrich Mann. Le poète et la politique, Klincksieck, Paris 1966).

Martine Benoit 